\title{
Ordinal-Measure Based Shape Correspondence
}

\author{
Faouzi Alaya Cheikh \\ Signal Processing Laboratory, Tampere University of Technology, P.O. Box 553, FIN-33101 Tampere, Finland \\ Email:faouzi@cs.tut.fi

\section{Bogdan Cramariuc} \\ Signal Processing Laboratory, Tampere University of Technology, P.O. Box 553, FIN-33101 Tampere, Finland \\ Email: crama@cs.tut.fi
}

\section{Mari Partio}

Signal Processing Laboratory, Tampere University of Technology, P.O. Box 553, FIN-33101 Tampere, Finland Email: partio@cs.tut.fi

\section{Pasi Reijonen}

Signal Processing Laboratory, Tampere University of Technology, P.O. Box 553, FIN-33101 Tampere, Finland Email: pasi.reijonen@tut.fi

\author{
Moncef Gabbouj \\ Signal Processing Laboratory, Tampere University of Technology, P.O. Box 553, FIN-33101 Tampere, Finland \\ Email: Moncef.Gabbouj@tut.fi
}

Received 31 July 2001 and in revised form 10 February 2002

\begin{abstract}
We present a novel approach to shape similarity estimation based on distance transformation and ordinal correlation. The proposed method operates in three steps: object alignment, contour to multilevel image transformation, and similarity evaluation. This approach is suitable for use in shape classification, content-based image retrieval, and performance evaluation of segmentation algorithms. The two latter applications are addressed in this paper. Simulation results show that in both applications our proposed measure performs quite well in quantifying shape similarity. The scores obtained using this technique reflect well the correspondence between object contours as humans perceive it.
\end{abstract}

Keywords and phrases: shape, ordinal, correlation, content, retrieval, indexing, segmentation, performance.

\section{INTRODUCTION}

Shape representation techniques are generally characterized as being boundary-based or region-based. The former (also known as contour-based) represents the shape by its outline, while the latter considers the shape as being formed of a set of two-dimensional regions. The human visual system itself focuses on edges and ignores uniform regions $[1,2]$. This capability is hardwired into the retina. Connected directly to the rods and cones of the retina are two layers of the neurons that perform an operation similar to the Laplacian. This operation is called lateral inhibition and helps us to extract boundaries and edges. Therefore, in this paper we focus on this aspect of the shapes and not on the regions they may contain. Object contours however, will have intrinsic intraclass variations. Moreover, object boundary deformation is expected in most imaging applications due to the varying imaging conditions, sensor noise, occlusion, and imperfect segmentation.

Estimating the similarity between objects shapes can be described in a simplistic way in two steps: shape features extraction and feature comparison. Each of these two steps however represents a difficult problem by itself. Selecting a set of features to characterize a shape for a certain application is not easy, since one must take into consideration the variability of the shapes and the application domain specificity. Feature comparison can be understood as a way of quantifying the similarity/dissimilarity between their corresponding objects. This is a very difficult problem since it tries to mimic the human perception [2].

Several shape features have been proposed in the literature for shape characterization [3]. Many of these techniques however, cannot be used for content-based indexing and retrieval due to their complexity or because they have no 


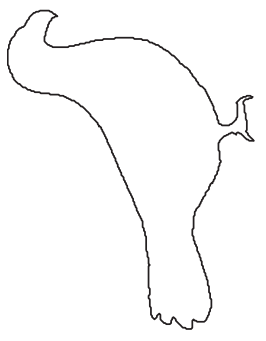

(a)

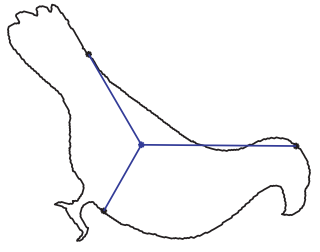

(b)
FIGURE 1: (a) The original bird contour before the alignment step, (b) the contour after alignment using three universal axes.

obvious counterpart in the human vision. Therefore, techniques based on simple and visually meaningful shape features have been used in several content-based indexing and retrieval (CBIR) systems, for example, QBIC [4], MUVIS $[5,6]$ such as high curvature points $[7,8,9]$, polygonal approximation [10], morphological and topological features and others $[3,11]$.

In this paper, we introduce a novel boundary-based approach to shape similarity estimation. This technique is applied to two problems: shape-based image retrieval and performance evaluation of segmentation algorithms. The rest of the paper is organized as follows: Section 2 presents an overview of the proposed method, followed by a detailed description of each step. Experimental results for both applications are presented in Section 3, using a subset of the MPEG-7 shape test data and the segmentation masks obtained by the COST Analysis Model (COST AM) [12, 13] segmentation algorithm. In Section 4, conclusions are drawn.

\section{THE PROPOSED METHOD}

Images in the target applications are representing either: a single object outline or a segmentation mask. Therefore, we will not discuss how to obtain the contour or the segmentation masks. Our goal is to compute a similarity score between any two shapes or two segmentation masks. The proposed method operates in three steps: alignment, boundary to multilevel image transformation, and similarity evaluation. The alignment step is not needed in the case of the segmentation performance evaluation, since we are comparing segmentations masks corresponding to the same image.

Once the boundaries are aligned, the binary images containing the boundaries are transformed into multilevel images through distance transformation [3]. The obtained images are then compared using the ordinal correlation measure introduced in $[14,15]$. This ordinal measure estimates the similarity between the two shapes based on the correlation of their corresponding transform images. In the rest of this section we give detailed description of each one of the steps mentioned above.

\subsection{Object alignment based on universal axes}

The alignment is performed by first detecting three universal axes [16] (those with the largest magnitude) for each shape, then orienting the shape in such a way that these axes are aligned in a standard way for all the objects to be compared. We use the same notation as in [16].

In this implementation of the universal axes determination algorithm we use the version number $\mu=2 l$. The steps of the alignment algorithm are detailed below.

Step 1. Translate the coordinate system so that the origin becomes the center of gravity of the shape $S$.

Step 2. Compute

$$
\begin{aligned}
\left|x_{\mu}^{(l)}+i y_{\mu}^{(l)}\right| & =\iint_{S}\left(\sqrt{x^{2}+y^{2}}\right)^{\mu}\left(\frac{x+i y}{\sqrt{x^{2}+y^{2}}}\right)^{l} d x d y \\
& =\iint_{S} r^{\mu} e^{i l \theta} d x d y
\end{aligned}
$$

and using normalized counterpart (called Universal Axes (UA))

$$
\left|\tilde{x}_{\mu}^{(l)}+i \tilde{y}_{\mu}^{(l)}\right|=\frac{\left|x_{\mu}^{(l)}+i y_{\mu}^{(l)}\right|}{\iint_{S}\left(\sqrt{x^{2}+y^{2}}\right)^{\mu} d x d y}, \quad \text { for } l=1,2,3 .
$$

Step 3. Compute the polar angle $\Theta_{\mu} \in[0,2 \pi]$ so that

$$
R_{\mu} e^{i \Theta_{\mu}}=\left|x_{\mu}^{\left(l_{1}\right)}+i y_{\mu}^{\left(l_{1}\right)}\right|
$$

with $R_{\mu}$ being the magnitude of $\left|x_{\mu}^{\left(l_{1}\right)}+i y_{\mu}^{\left(l_{1}\right)}\right| . l_{1}$ is the number of axes needed to align an object.

Step 4. Compute the directional angles of the $l_{1}$ universal axes of the shape $S$ as follows:

$$
\theta_{j}=\frac{\Theta_{\mu}}{l_{1}}+(j-1) \frac{2 \pi}{l_{1}}, \quad \text { for } j=1,2, \ldots, l_{1} .
$$

In our implementation we used $l_{1}=3$, see Figure 1 , since for $l_{1}=2$ the two universal axes orientation will verify $\theta_{2}=$ $\theta_{1}+\pi$. Therefore, they cannot be used alone to determine if an object is flipped around the direction they define or not.

Step 5. Once the three universal axes are determined, rotate the contour so that the most dominant UA (UA with the largest magnitude) will be aligned with the positive $x$-axis, see Figure 1.

Step 6. Then, if the $y$-component of the second most dominant UA is positive, flip the contour around the $x$-axis.

To illustrate the alignment performance we applied it to the set of contours in Figure 2. The results of the alignment are presented in Figure 3. It can be noticed that this alignment scheme solved both problems of rotation and mirroring. 


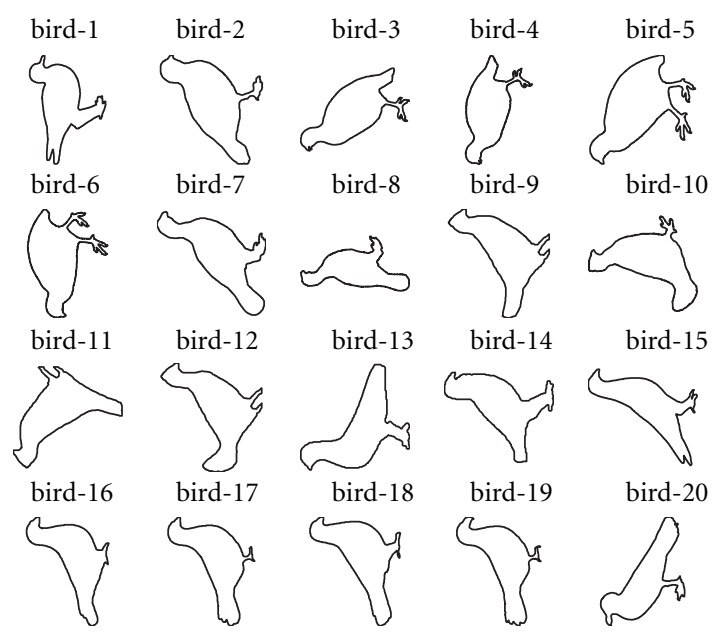

FIgURe 2: Bird contours from the MPEG-7 shape test set B.

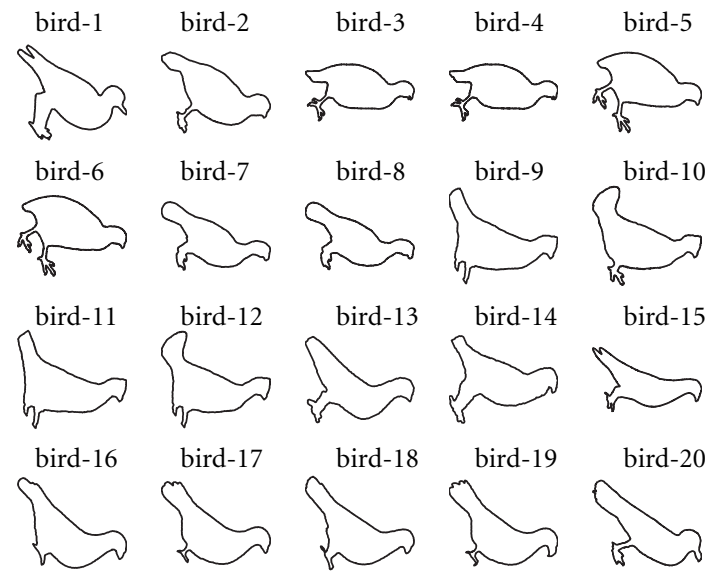

FIgURE 3: The contours in Figure 2 after alignment.

\subsection{Boundary to multilevel image transformation}

Let $S$ be a shape represented by its contour $C$ in a binary image. The binary image is transformed into a multilevel (grayscale) image $G$ using a mapping function $\phi$, such that the pixel values in $G,\left\{G_{1}, G_{2}, \ldots, G_{n}\right\}$, depend on their relative position to the contour pixels $C_{1}, C_{2}, \ldots, C_{p}$ :

$$
G_{i}=\phi\left(C_{k}: k=1,2, \ldots, p\right), \quad \text { for } i=1,2, \ldots, n,
$$

where $C_{k}$ is the position of the contour pixel $k$ in the image $G$. It should be observed that several transformations satisfy this requirement, including any distance transform [3].

As a result of this mapping the information contained in the shape boundary will be spread throughout all the pixels of the image. Computing the similarity in the transform domain will benefit from the boundary information redundancy in the new image. We expect that there is no single optimal mapping; different mappings will emphasize different features of the contour. We distinguish however two special cases:
- the first is defined as follows:

$$
G_{i}= \begin{cases}V_{0}-d\left(P_{i}, C\right), & \text { if } d\left(P_{i}, C\right)<T_{h}, \\ 0, & \text { otherwise, }\end{cases}
$$

for $i=1,2, \ldots, n$ and $V_{0}>0$ is the value assigned to the pixels on the boundary. The larger the distance $d\left(P_{i}, C\right)$ from the contour points is, the smaller the new pixel value will be. This mapping function emphasizes the details on the boundary;

- the second mapping is when $V_{0}=0$ and $G_{i}=d\left(P_{i}, C\right)$, using a geodesic distance [17]. When this mapping is applied inside the contour only, the emphasis is on the shape skeleton which is a very important feature of a shape, see Figure 4. Applying the distance mapping inside and outside the contour can lead to a better evaluation of segmentation results. One can even assign different weights inside and outside of the contours.

In this work, we implemented the second mapping based on the geodesic distance. The metric is integer and its application is done through an iterative wave propagation process [18]. The contour points are considered as seeds during the construction of the distance map. The distance map can be generated inside and/or outside the contour, as stated earlier. The values can increase or decrease starting from the contour and can be limited. The pixel values in the distance map can be therefore written as follows:

$$
G_{i}=\left|V_{0} \pm d\left(P_{i}, C\right)\right|, \quad \text { for } i=1,2, \ldots, n,
$$

where, $V_{0}$ is the value on the contour and $d\left(P_{i}, C\right)$ is the distance from any point $P_{i}$ in the image to the contour $C$.

Figure 4a represents an example of a distance map generated only inside the contour of a bird contour. Figure $4 \mathrm{~b}$ shows a $3 \mathrm{D}$ visualization of this distance map.

\subsubsection{Similarity evaluation}

The evaluation of image similarity is based on the framework for ordinal-based image correspondence introduced in [14]. Figure 5 gives a general overview of this region-based approach.

Suppose we have two images, $X$ and $Y$, of equal size. In a practical setting, images are resized to a common size. Let $\left\{X_{1}, X_{2}, \ldots, X_{n}\right\}$ and $\left\{Y_{1}, Y_{2}, \ldots, Y_{n}\right\}$ be the pixels of images $X$ and $Y$, respectively. We select a number of areas $\left\{R_{1}, R_{2}, \ldots, R_{m}\right\}$ and extract the pixels from both images that belong to these areas. Let $R_{j}^{X}$ and $R_{j}^{Y}$ be the pixels from images $X$ and $Y$, respectively, which belong to areas $R_{j}$, with $j=1,2, \ldots, m$.

The goal is to compare the two images using a regionbased approach. To this end, we will be comparing $R_{j}^{X}$ and $R_{j}^{Y}$ for each $j=1,2, \ldots, m$. Thus, each block in image $X$ is compared to the corresponding block in image $Y$ in an ordinal fashion. The ordinal comparison of the two regions means that only the ranks of the pixels are utilized. For every pixel $X_{k}$, we construct a so-called slice $S_{k}^{X}=\left\{S_{k, l}: l=1,2, \ldots, n\right\}$, 


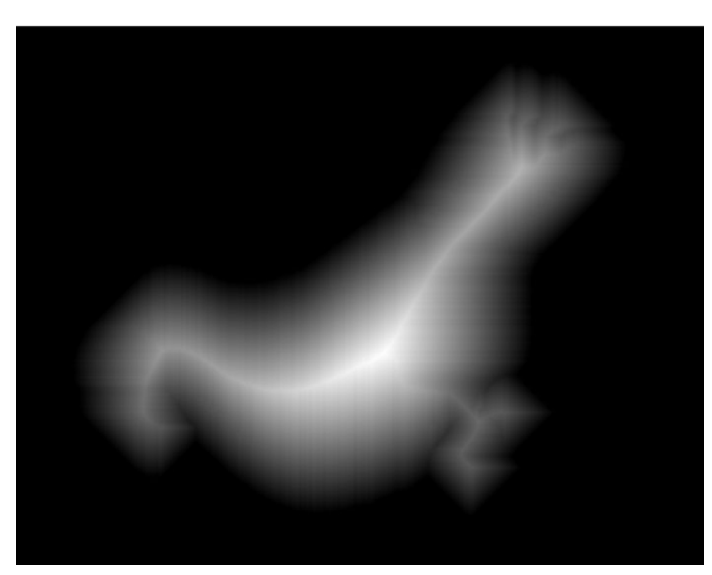

(a)

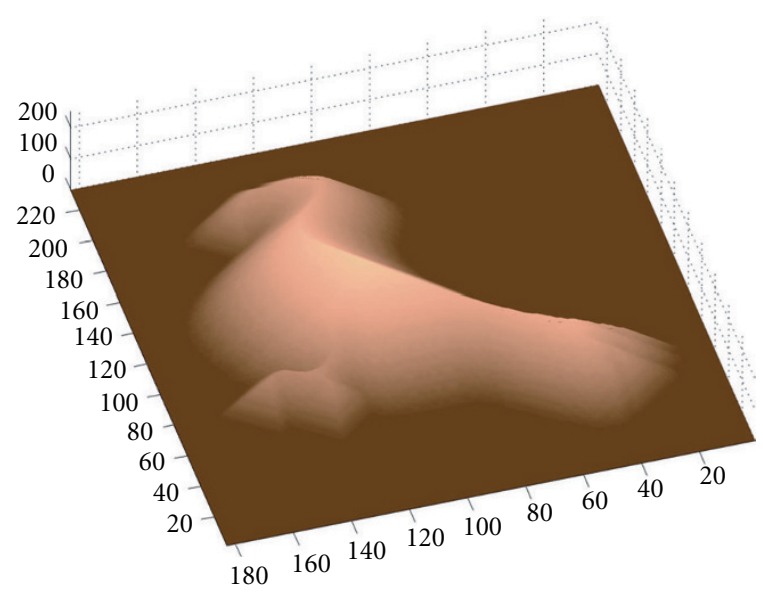

(b)

Figure 4: (a) The distance map generated for the "bird-19" contour in Figure 1, (b) a 3D view of this distance map.

where

$$
S_{k, l}^{X}= \begin{cases}1, & \text { if } X_{k}<X_{l} \\ 0, & \text { otherwise }\end{cases}
$$

As can be seen, slice $S_{k}^{X}$ corresponds to pixel $X_{k}$ and is a binary image of size equal to image $X$. Slices are built in a similar manner for image $Y$ as well.

To compare regions $R_{j}^{X}$ and $R_{j}^{Y}$, we first combine the slices from image $X$, corresponding to all the pixels belonging to region $R_{j}^{X}$. The slices are combined using the operation $\mathrm{OP}_{1}(\cdot)$ into a metaslice $M_{j}^{X}$.

Figure 6 shows an illustration of the slices and metaslices creation for a $4 \times 4$ image and blocks of $2 \times 2$. The four slices $S_{1}, S_{2}, S_{5}$, and $S_{6}$ shown in this figure are computed for the four pixels in block $B_{1}$. The operation used in this illustration to create the metaslice $M_{1}$ is $\mathrm{OP}_{1}(\cdot)=\sum(\cdot)$.

More formally, $M_{j}^{X}=\mathrm{OP}_{1}\left(S_{k}^{X}: X_{k} \in R_{j}^{X}\right)$ for $j=$ $1,2, \ldots, m$. Similarly, we combine the slices from image $Y$ to form $M_{j}^{Y}$ for $j=1,2, \ldots, m$. It should be noted that the metaslices are equal in size to the original images and could be multivalued, depending on the operation $\mathrm{OP}_{1}(\cdot)$. Each metaslice represents the relation between the region it corresponds to and the entire image.

The next step is a comparison between all pairs of metaslices $M_{j}^{X}$ and $M_{j}^{Y}$ by using operation $\mathrm{OP}_{2}$, resulting in the metadifference $D_{j}$. That is, $D_{j}=\mathrm{OP}_{2}\left(M_{j}^{X}, M_{j}^{Y}\right), j=$ $1,2, \ldots, m$. We thus construct a set of metadifferences $D=$ $\{D 1, D 2, \ldots, D m\}$. The final step is to extract a scalar measure of correspondence from set $D$, using operation $\mathrm{OP}_{3}(\cdot)$. In other words, $\lambda=\mathrm{OP}_{3}(D)$. It was shown in [14] that this structure could be used to model the well-known Kendall's $\tau$ and Spearman's $\rho$ measures [19].

The image similarity measure used in this paper is an instance of the previously mentioned framework. This measure has been analyzed more extensively by Cramariuc et al. [15]. Following is a short description of the operations $\mathrm{OP}_{k}(\cdot), k=1,2,3$ adopted for this measure. Operation $\mathrm{OP}_{1}(\cdot)$ is chosen to be the component-wise summation operation; that is, metaslice $M_{j}$ is the summation of all slices corresponding to the pixels in block $j$ or in other words, $M_{j}=\sum_{k: X_{k} \in R_{j}} S_{k}$.

Next, operation $\mathrm{OP}_{2}(\cdot)$ is chosen to be the squared Euclidean distance between corresponding metaslices. That is, $D_{j}=\left\|M_{j}^{X}-M_{j}^{Y}\right\|_{2}^{2}$. Finally, operation $\mathrm{OP}_{3}(\cdot)$ sums together all metadifferences to produce $\lambda=\sum_{j} D_{j}$, for $j=1,2, \ldots, m$. Small values of $\lambda$ mean similar objects.

One advantage of this approach over classical ordinal correlation measures is its capability to take into account differences between images at a scale related to the chosen block size.

\section{EXPERIMENTAL RESULTS}

The proposed technique is applied to two important problems: content-based retrieval of shape images and performance evaluation of segmentation algorithms. The experiments performed are presented and their results analyzed in the rest of this section.

\subsection{Shape similarity estimation}

The shape similarity estimation experiments were conducted on two sets of 20 images. The two sets are taken from the MPEG-7 CE Shape test set B, which contains 1400 images grouped in 70 categories. These test sets are chosen in such a way as to assess the performance of our technique in estimating the object similarity within a single category (intracategory) and between contours from different categories (inter-category). Therefore, the first test set contains all the samples in the bird category of the MPEG-7 Shape test set B, see Figure 2. While, the second set contains 20 objects taken from four different categories, see Figure 7. In both experiments, the similarity score $\lambda$ is computed for all the pairs 


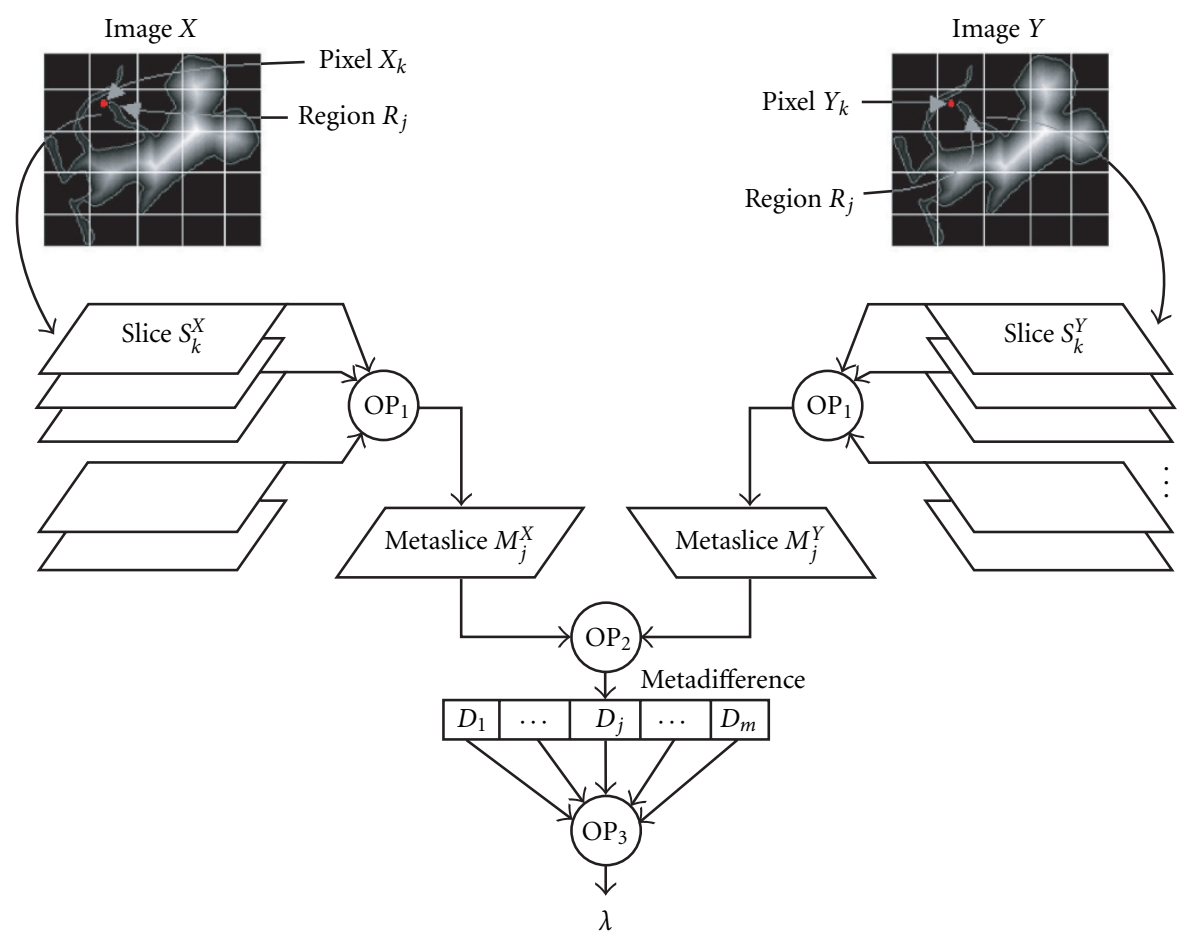

FIGURE 5: The general framework for ordinal correlation of images.
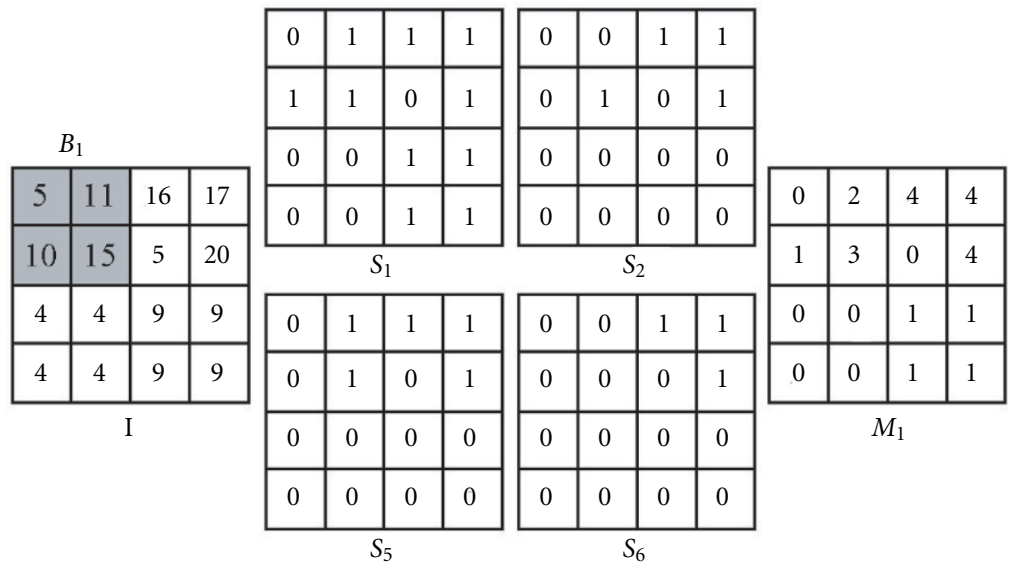

FIgURE 6: Example of slices and metaslice for a $4 \times 4$ image using blocks of $2 \times 2$.

of shapes in the set. The similarity scores obtained are presented in Tables 1 and 2 . All the scores are multiplied by $10^{3}$ when they are presented in the tables and the figures. The distance maps were generated inside the objects only with $V_{0}=50$. This setting emphasizes the shape skeleton and gives less importance to contour pixels. The distance transformed images are resized to $32 \times 32$ pixels and blocks of size $4 \times 4$ were used. Larger images can be used if more precision is needed, this would imply the creation of more slices and therefore more computational power would be needed.

Figure 8 represents a surface plot of the similarity scores in Table 1. It shows that within the same category, the scores have small values, which means that they are quite similar according to our measure. It is worth noticing that the scores on the diagonals are zero which means that each object is identical to itself, so there is no bias in the similarity scores. It is worth noticing that the scores obtained between the "bird3," "bird-4," "bird-5," "bird-6," and the rest of the birds in this category are larger than the rest of the scores. This can be explained by the fact that these four birds have much shorter tails and have a more circular contours compared the rest of the birds. The similarity scores are low between themselves, moreover, the scores for the pairs (bird-3, bird-4), (bird-5, bird-6), (bird-7, bird-8), and (bird-9, bird-11) are very small. By visual inspection one can verify that each pair of con- 
TABLE 1: Similarity scores for the contours in Figure 3. These scores are multiplied by $10^{3}$.

\begin{tabular}{|c|c|c|c|c|c|c|c|c|c|c|c|c|c|c|c|c|c|c|c|c|}
\hline jject & 竭 & 汸 & 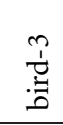 & 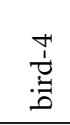 & 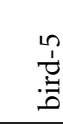 & 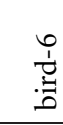 & $\begin{array}{l}\hat{1} \\
0 \\
0 \\
0\end{array}$ & : & 竞 & $\begin{array}{l}0 \\
1 \\
.0 \\
0 \\
0\end{array}$ & $\begin{array}{l}\overline{7} \\
\overline{0} \\
\overrightarrow{0}\end{array}$ & $\stackrel{7}{7}$ & $\begin{array}{l}0 \\
\frac{1}{3} \\
0 \\
0\end{array}$ & 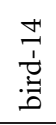 & $\begin{array}{l}n \\
0 \\
0 \\
0 \\
0\end{array}$ & 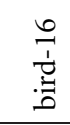 & $\begin{array}{l}\overline{7} \\
\overline{1} \\
0\end{array}$ & 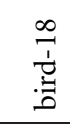 & 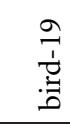 & $\underset{T}{1}$ \\
\hline rd-1 & 0 & 32 & 75 & 74 & 80 & 81 & 46 & 44 & 52 & 44 & 52 & 49 & 64 & 49 & 53 & 63 & 60 & 51 & 64 & \\
\hline $\mathrm{rd}-2$ & 32 & 0 & 71 & 70 & 79 & 79 & 39 & & 60 & 59 & 62 & 61 & 65 & 56 & 53 & 68 & 58 & 52 & 62 & \\
\hline $\mathrm{rd}-3$ & 75 & 71 & 0 & 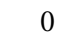 & 48 & 48 & 80 & 7 & 88 & 84 & & 87 & 102 & 53 & 7 & & 12 & 105 & 116 & \\
\hline rd & 74 & 70 & 0 & 0 & 48 & 48 & 80 & 76 & 88 & 83 & 85 & 87 & 02 & 53 & 71 & & 12 & 04 & 16 & \\
\hline 5 & 80 & 79 & 48 & 48 & 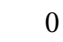 & 1 & 101 & 98 & 105 & 89 & 2 & 98 & 120 & 76 & 95 & 25 & 22 & 12 & 25 & 2 \\
\hline rd-6 & 81 & 79 & 48 & 48 & 1 & 0 & 101 & 98 & 106 & 90 & 100 & 99 & 120 & 16 & 95 & 120 & 122 & 113 & 120 & 21 \\
\hline $\mathrm{rd} 7$ & 46 & 39 & 80 & 80 & 101 & 101 & 0 & & 39 & 42 & 42 & 40 & 42 & 54 & 28 & 53 & (1) & 38 & & \\
\hline 8 & 4 & 38 & 77 & 7 & 98 & 98 & 7 & 0 & 38 & 39 & נת נJ & & 44 & 52 & 2 & & 4 & 41 & 51 & \\
\hline d-9 & 52 & 60 & 88 & 8 & 105 & 106 & 39 & 38 & 0 & 25 & 7 & 17 & 32 & 51 & JJ & & 4 & 39 & 49 & \\
\hline d- -10 & 44 & 59 & 84 & 83 & 89 & 90 & 42 & 3 & 25 & . & 22 & 12 & 45 & 53 & 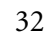 & 5 & 48 & 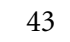 & & \\
\hline 1 & 52 & 62 & 85 & 8 & 102 & 103 & 4 & & 7 & 2 & & & 3 & 1 & & & & 45 & & \\
\hline d-12 & 49 & 61 & 87 & 8 & 98 & 99 & 40 & 38 & 17 & 1 & 10 & 0 & 37 & 55 & 31 & 5 & 46 & 41 & 50 & \\
\hline 13 & 64 & 65 & 102 & & 120 & 120 & 42 & 4 & 32 & 45 & & רב & & 60 & 42 & & & 37 & & \\
\hline 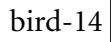 & 4 & 5 & 5 & 5 & 76 & 76 & & & & 5 & 49 & & 60 & 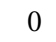 & 47 & & & & & \\
\hline d-15 & 53 & 53 & 71 & 7 & 95 & 95 & 28 & 2 & 33 & 32 & 31 & 31 & 42 & 47 & ( & 67 & 56 & 52 & 59 & \\
\hline & 63 & 68 & & & - & 6 & 53 & J & & 55 & & 52 & & 86 & 67 & & 17 & 22 & 19 & \\
\hline & 60 & 5 & & & & 12 & 4 & 4 & & 4 & 1 & 46 & & 80 & 5 & 1 & & 10 & & \\
\hline 8 & 51 & 52 & 1 & 1 & 112 & 11 & r & 4 & 39 & 4 & 45 & 4 & 37 & 75 & 5 & 2 & 18 & 0 & 17 & \\
\hline & 64 & 62 & 11 & 11 & - & 126 & 4 & & 4 & 53 & & 5 & 40 & 86 & & 1 & & 17 & & \\
\hline $\mathrm{rd}-20$ & 5 & 55 & 106 & 106 & 121 & 121 & 43 & 47 & 60 & 60 & 63 & 61 & 87 & 83 & 61 & 44 & 39 & 35 & 38 & \\
\hline
\end{tabular}

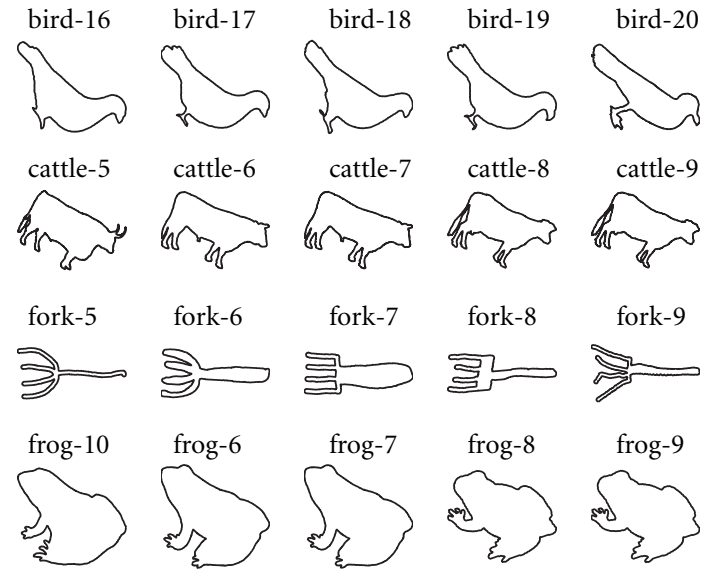

Figure 7: Contours of test set 2 after alignment.

tours represent the same bird contour rotated or rotated and scaled. Therefore, we can safely say that our measures have a $0.5 \%$ error, which can be explained by the small contour variation introduced by rotation and the size reduction of the distance maps. Lower error can be obtained by increasing the size of the distance map images and reducing the block size used for the metaslices creation.

Dark blue regions in Figure 8 represent very low scores (close to zero), which shows that there are quite many objects in this category which are very similar or even identical.

To find out which are the most similar contours to a given contour in Figure 7 we sort the scores on the raw corresponding to this contour in Table 2. Using Figure 9, one can easily

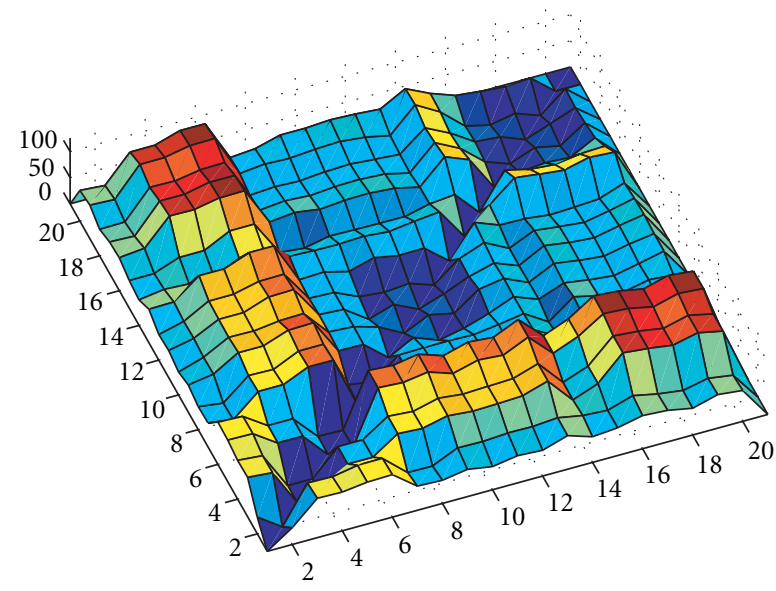

Figure 8: The similarity scores for the bird contours in Figure 3, dark blue cells mean most similar contours.

estimate which are the most similar objects within this category, based on the clustered dark blue cells. Figure 9 shows that similarity scores between subjects from the same category are low, while those obtained for subjects from different categories are relatively high. Therefore, sorting the scores in ascending order will yield the most similar object first.

The inter-category scores obtained by our similarity estimation technique are larger than the inter-category ones. Therefore, this technique can be used as a shape classification technique. Moreover, it is sensitive to intra-category shape variations thus it can be used in a content-based retrieval system. 
TABLE 2: Similarity scores for the contours in Figure 7. These scores are multiplied by $10^{3}$.

\begin{tabular}{|c|c|c|c|c|c|c|c|c|c|c|c|c|c|c|c|c|c|c|c|c|}
\hline . & $\begin{array}{l}0 \\
1 \\
\end{array}$ & 竞 & $\stackrel{\infty}{\longrightarrow}$ & $\begin{array}{l}a \\
\frac{1}{2} \\
.7 \\
0\end{array}$ & 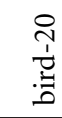 & 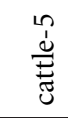 & 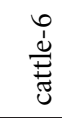 & 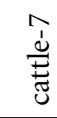 & $\frac{\dot{d}}{\stackrel{0}{\rightleftarrows}}$ & 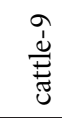 & $\begin{array}{l}\frac{10}{4} \\
\frac{1}{0}\end{array}$ & 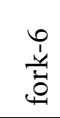 & $\frac{1}{\tilde{0}}$ & $\begin{array}{l}\infty \\
\dot{1} \\
\\
0\end{array}$ & $\frac{a}{\dot{y}}$ & $\begin{array}{l}0 \\
0 \\
0 \\
0 \\
0\end{array}$ & 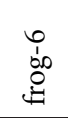 & مَ & 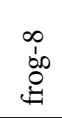 & فे \\
\hline 5 & 0 & 39 & 63 & 39 & 101 & 282 & 322 & 321 & 351 & 348 & 58 & 368 & 361 & 379 & 401 & 196 & 209 & 210 & 303 & 30 \\
\hline & 39 & 0 & 58 & 22 & 94 & 3 & P & 4 & 342 & 341 & 7 & 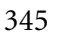 & 339 & 363 & 377 & 99 & 206 & 08 & 297 & 29 \\
\hline & 63 & 58 & & 58 & 83 & 6 & & 1 & & & & & 3 & & 4 & 32 & & & & \\
\hline & 39 & 22 & 58 & 0 & 74 & 88 & 2 & 331 & 369 & 366 & 60 & 68 & 63 & 388 & 402 & 215 & 27 & 28 & 18 & 31 \\
\hline rd-20 & 01 & 94 & 83 & 74 & 0 & 304 & 33 & 334 & 370 & 367 & 5 & 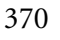 & 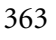 & 1 & 2 & 234 & 233 & 234 & 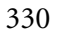 & 330 \\
\hline & 2 & 73 & 256 & 298 & 304 & 0 & 73 & 71 & 75 & 73 & 7 & L & & & 14 & & & & 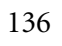 & 138 \\
\hline & 2 & 6 & 293 & 32 & 333 & 73 & 0 & 1 & 52 & 53 & 46 & 04 & 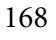 & . & 234 & 1 & 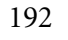 & 8 & 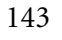 & 145 \\
\hline 7 & 21 & 304 & 2 & 11 & 334 & 71 & 1 & 0 & 51 & 52 & 47 & 206 & 169 & 1 & 236 & & 91 & 2 & & 14 \\
\hline & & & 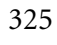 & 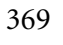 & 370 & 75 & 52 & 5 & & & 244 & 207 & 172 & 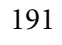 & 228 & 2 & 199 & 200 & & 143 \\
\hline & 48 & 1 & 322 & 366 & 367 & 73 & 53 & 52 & 5 & 0 & 244 & 209 & 173 & 190 & 232 & 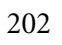 & 96 & 197 & , & 142 \\
\hline 5 & 358 & 337 & 325 & 360 & 365 & 247 & 246 & 247 & 244 & 244 & 0 & 59 & 118 & 84 & 63 & 373 & 347 & 346 & 3 & 35 \\
\hline & 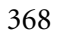 & 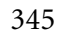 & 3 & 368 & 370 & 22 & 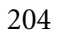 & 206 & 20 & 20 & & . & 62 & 14 & 77 & 355 & 337 & 338 & 32 & 32 \\
\hline & 61 & 9 & 323 & 3 & 63 & 184 & & 169 & 172 & 173 & 118 & 62 & 0 & 81 & 123 & 20 & 304 & 305 & 287 & 290 \\
\hline & 379 & 3 & & & 387 & 206 & & 199 & 19 & 19 & 8 & 74 & 81 & 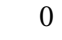 & 79 & 81 & 331 & 331 & 319 & 321 \\
\hline & & & 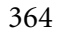 & 402 & 392 & 244 & & 236 & 22 & 23 & 63 & 77 & 123 & 79 & 0 & 379 & 354 & 352 & 339 & 340 \\
\hline 10 & 96 & 199 & 18 & 15 & 234 & 162 & 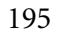 & 193 & 206 & 202 & 373 & 355 & 320 & 351 & 379 & & 50 & 51 & 132 & 129 \\
\hline & 209 & 6 & & 27 & 33 & 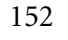 & & 91 & 199 & 196 & 347 & 337 & 304 & 331 & 354 & 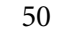 & 0 & & 138 & 138 \\
\hline & 21 & 8 & r & 228 & 234 & 153 & & 192 & 20 & 197 & 346 & 338 & 305 & 331 & 352 & 31 & 1 & 0 & 139 & 139 \\
\hline & 305 & & 20 & 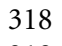 & 330 & 10 & & 14 & 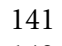 & 140 & 35 & 326 & 287 & 319 & 339 & 132 & 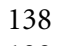 & 139 & & \\
\hline g-9 & 302 & 297 & 284 & 318 & 330 & 138 & 15 & 144 & 143 & 142 & 359 & 329 & 290 & 321 & 340 & 129 & 138 & 139 & 2 & \\
\hline
\end{tabular}

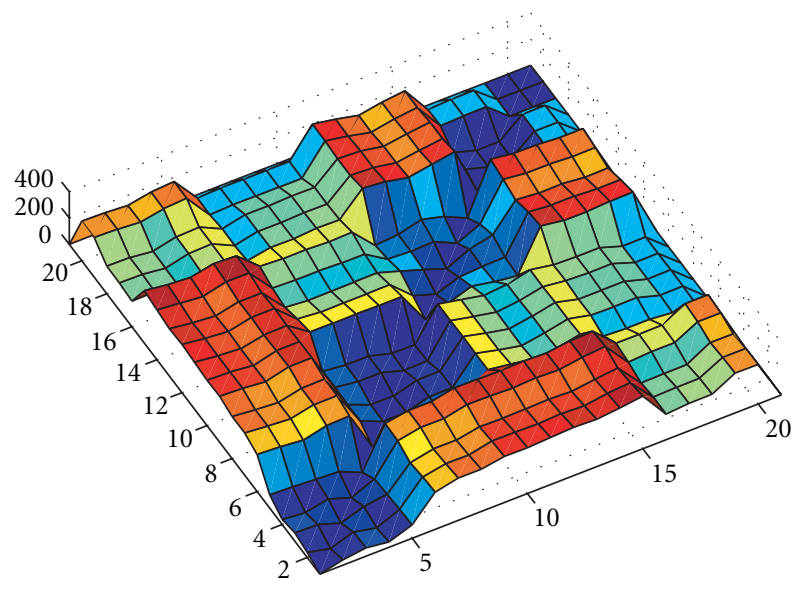

FIGURE 9: Similarity scores obtained for the contours in test set 2 presented in Figure 7.

\subsection{Segmentation quality evaluation}

The objective evaluation of the performance of segmentation algorithms is an important problem [20,21, 22]. Even when a reference mask is available, comparing two segmentation masks is still a difficult problem. Several factors make such evaluation difficult, among the most important factors is the difficulty to discriminate between many small distributed error segments and few larger error segments.

Our shape correspondence technique proposed in Section 2, discriminates easily between the two cases of segmentation errors. The geodesic distance transformation is applied inside each segment of the mask. Therefore, small regions yield small distances inside them and therefore will generate pixels with low gray values.

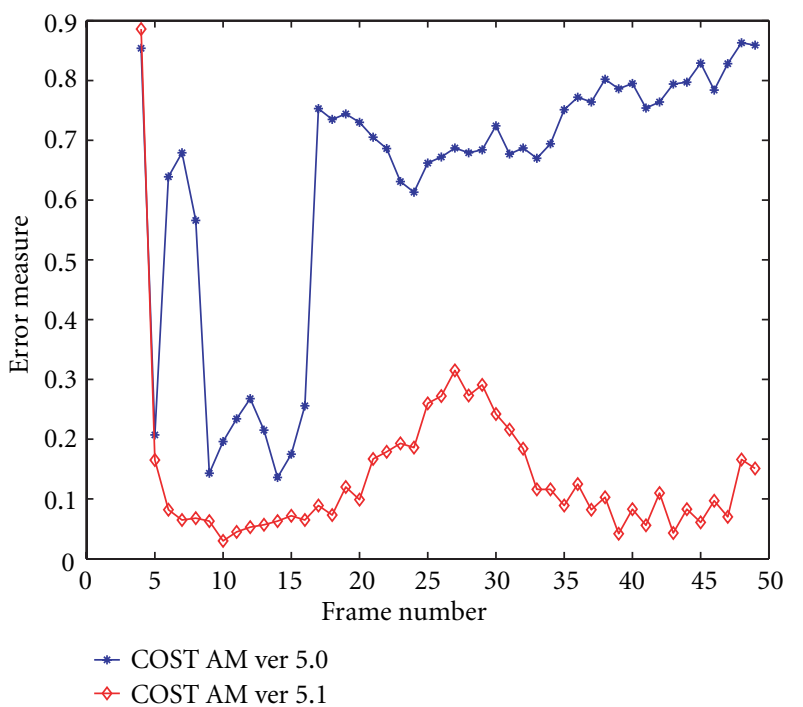

FIGURE 10: The segmentation performance scores for frames 4-49 of the sequence "Erik," for COST AM versions 5.0 and 5.1.

In this experiment, the segmentation masks resulting from the COST AM versions 5.0 and 5.1 [12, 13], are compared against a reference mask. The plot in Figure 10, shows the segmentation performance scores obtained by our technique, for the frames 4-49 of "Erik" sequence. The plots in Figures 11 and 12, show quantitative measures of the errors in number of pixels from both COST AM versions 5.0 and 5.1, respectively. Three different numbers are computed for each frame:

- number of pixels of the background segmented as foreground pixels, 


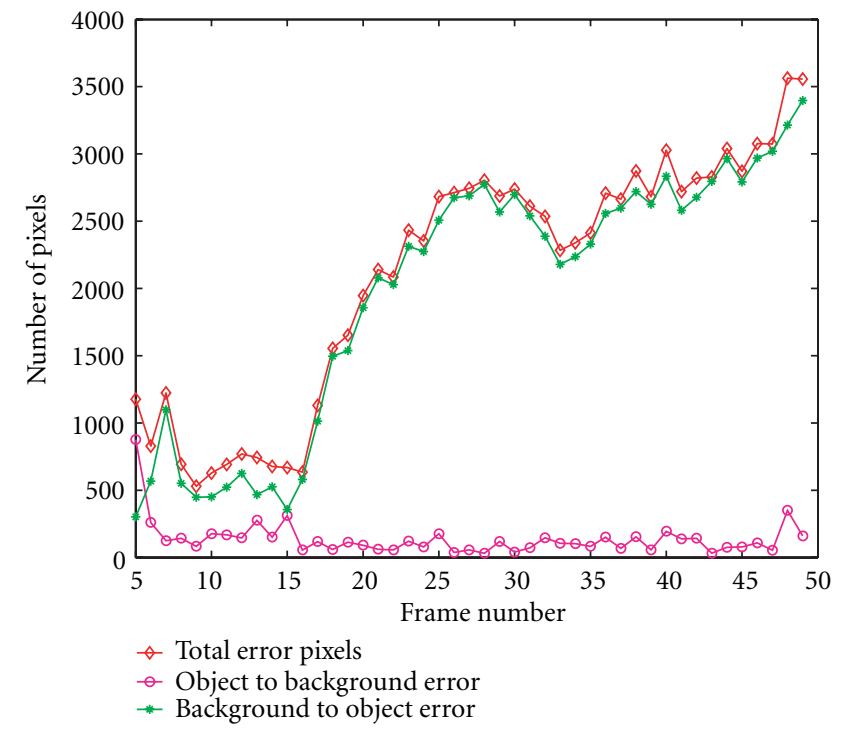

Figure 11: Plot of the segmentation errors, for COST AM 5.0.

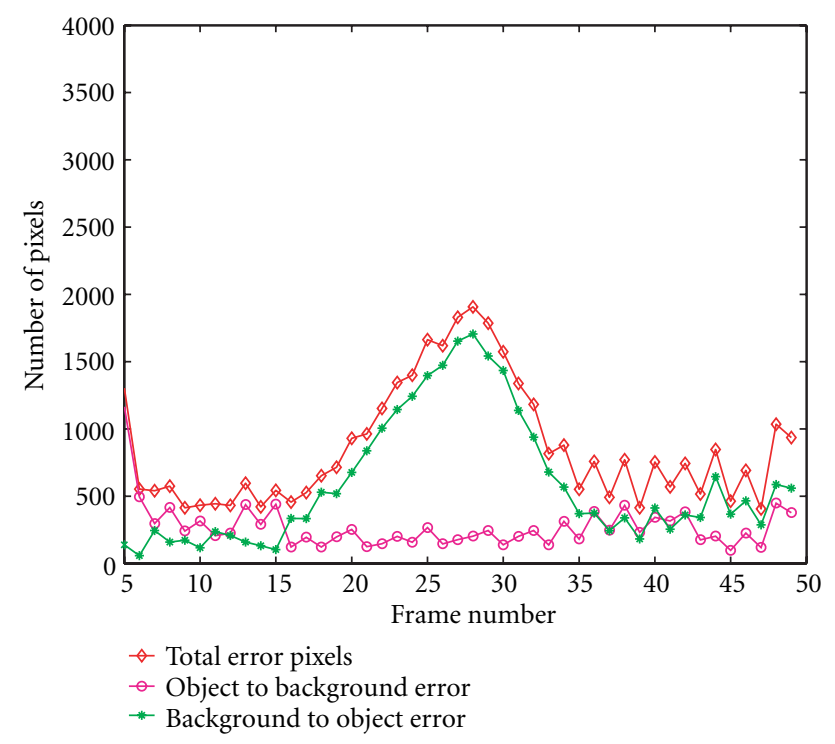

Figure 12: Plot of the segmentation errors, for COST AM 5.1.

- number of pixels of the foreground segmented as background pixels,

- sum of the two previous numbers.

For illustration we present the colored segmentation masks for frames 15 and 20 from "Erik" sequence in Figures $13,14,15$, and 16. The frame pixels are colored as follows:

- black represents the background,

- white is the region where the reference and estimated masks overlap,

- green represents the areas of the background segmented as part of the object,

- purple represents the regions from the object merged with the background.

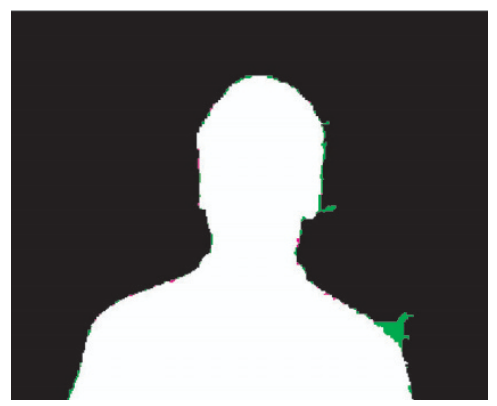

FIGURE 13: The colored segmentation error of frame 15 from the sequence "Erik," segmented using COST AM 5.0.

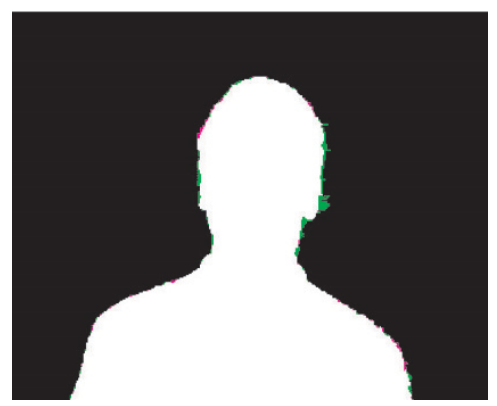

FIGURE 14: The colored segmentation result of frame 15 from the sequence "Erik," segmented using COST AM 5.1.

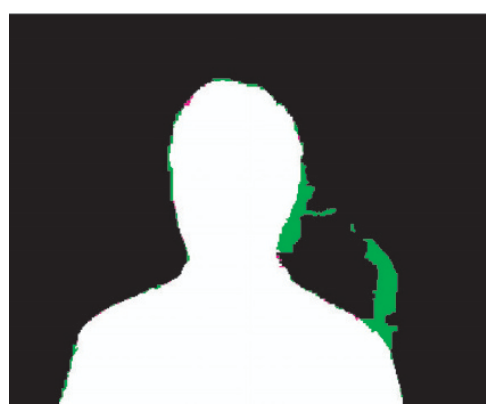

FigURE 15: The colored segmentation result of frame 20 from the sequence "Erik," segmented using COST AM 5.0.

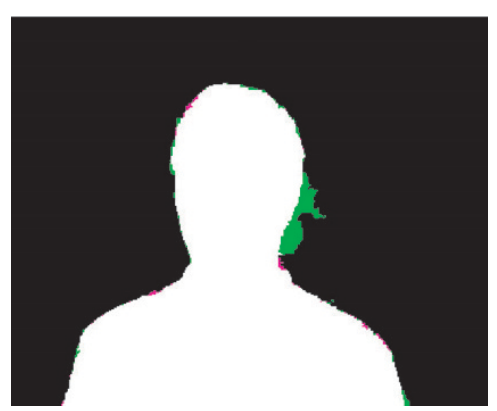

FIGURE 16: The colored segmentation result of frame 20 from the sequence "Erik," segmented using COST AM 5.1. 
It can be easily seen that our segmentation performance scores in Figure 10, correlate very well with the variation of the total number of pixel errors. Moreover, it reflects the variation in both types of segmentation errors. Our measure inherently resolves the case of many small errors and the case of a single large error region.

\section{CONCLUSIONS AND FUTURE WORK}

In this paper, we proposed a contour correspondence measure, based on distance transformation and ordinal correlation. The similarity scores obtained are in line with the visual perception of the similarity between shapes. We showed that the proposed measure can be used in two applications: shape similarity estimation in the context of content-based image retrieval, and for performance evaluation of segmentation algorithms. Simulation results were presented for both applications using images from the MPEG-7 shape test set B for the first application, and 50 frames from "Erik" sequence for the second. The proposed technique produced encouraging results in both experiments. Further study is needed to optimize the proposed technique in order to select appropriate parameters for the application at hand. Finally, further analysis of the behavior of the proposed technique may identify new applications.

\section{REFERENCES}

[1] C. Hildreth, "The detection of intensity changes by computer and biological vision systems," in Proc. Computer Vision, Graphics, and Image Processing, vol. 22, pp. 1-27, 1983.

[2] T. V. Papathomas, "Special issue on visual-perception: guest editorial," International Journal of Imaging Systems and Technology, vol. 7, no. 2, pp. 63-64, 1996.

[3] L. da F. Costa and R. M. Cesar Jr., Shape Analysis and Classification: Theory and Practice, CRC Press, Boca Raton, Fla, USA, 2001.

[4] M. Flickner, H. Sawhney, W. Niblack, et al., "Query by image and video content: The qbic system," IEEE Computer Magazine, vol. 28, no. 9, pp. 23-32, 1995.

[5] C. F. Alaya, B. Cramariuc, C. Reynaud, et al., "Muvis: a system for content-based indexing and retrieval in large image databases," in Proc. SPIE/EI '99 Conference on Storage and Retrieval for Image and Video Databases VII, vol. 3656, pp. 98106, San Jose, Calif, USA, January 1999.

[6] M. Trimeche, C. F. Alaya, M. Gabbouj, and B. Cramariuc, "Content-based description of images for retrieval in large databases: Muvis," in Proc. X European Signal Processing Conference, Tampere, Finland, September 2000.

[7] C. H. Teh and R. T. Chin, "On the detection of dominant points on digital curves," IEEE Trans. on Pattern Analysis and Machine Intelligence, vol. 11, no. 8, pp. 859-872, 1989.

[8] S. Abbasi, F. Mokhtarian, and J. Kittler, "Curvature scale space image in shape similarity retrieval," Springer Journal of Multimedia Systems, vol. 7, no. 6, pp. 467-476, 1999.

[9] A. Quddus, C. F. Alaya, and M. Gabbouj, "Wavelet-based multi-level object retrieval in contour images," in Proc. International Workshop on Very Low Bit Rate Video Coding, pp. 1-5, Kyoto, Japan, October 1999.

[10] M. W. Koch and R. L. Kashyap, "Using polygon to recognize and locate partially occluded objects," IEEE Trans. on Pattern Analysis and Machine Intelligence, vol. 9, no. 4, pp. 483-494, 1987.
[11] J. C. Russ, The Image Processing Handbook, CRC, Springer and IEEE Press, 3rd edition, 1999.

[12] A. Alatan, L. Onural, M. Wollborn, R. Mech, E. Tuncel, and T. Sikora, "Image sequence analysis for emerging interactive multimedia services-the European COST 211 framework," IEEE Trans. Circuits and Systems for Video Technology, vol. 8, no. 7, pp. 802-813, 1998.

[13] M. Gabbouj, G. Morrison, C. F. Alaya, and R. Mech, "Redundancy reducation techniques and content analysis for multimedia services-the European COST 211quat action," in Proc. Workshop on Image Analysis for Multimedia Interactive Services, Berlin, Germany, 31 May-1 June 1999.

[14] I. Shmulevich, B. Cramariuc, and M. Gabbouj, "A framework for ordinal-based image correspondence," in Proc. X European Signal Processing Conference, Tampere, Finland, September 2000.

[15] B. Cramariuc, I. Shmulevich, M. Gabbouj, and A. Makela, "A new image similarity measure based on ordinal correlation," in Proc. International Conference on Image Processing, vol. 3, pp. 718-721, Vancouver, BC, Canada, September 2000.

[16] J. C. Lin, "The family of universal axes," Pattern Recognition, vol. 29, no. 3, pp. 477-485, 1996.

[17] P. J. Toivanen, "New geodesic distance transforms for grayscale images," Pattern Recognition Letters, vol. 17, no. 5, pp. 437-450, 1996.

[18] I. Ragnelmam, "Neighborhoods for distance transformations using ordered propagation," Computer Vision, Graphics and Image Processing, vol. 56, no. 3, pp. 399-409, 1992.

[19] M. Kendall and J. D. Gibbons, Rank Correlation Methods, Edward Arnold, New York, 5th edition, 1990.

[20] P. Villegas, X. Marichal, and A. Salcedo, "Objective evaluation of segmentation masks in video sequences," in Proc. Workshop on Image Analysis for Multimedia Services (WIAMIS '1999), Berlin, Germany, May 1999.

[21] R. Mech, "Objective evaluation criteria for 2D-shape estimation results of moving objects," in Proc. Workshop on Image Analysis for Multimedia Services, pp. 23-28, Tampere, Finland, May 2001.

[22] R. Mech and F. Marqu s, "Objective evaluation criteria for 2Dshape estimation results of moving objects," EURASIP Journal of Applied Signal Processing, Special Issue: Image Analysis for Multimedia Interactive Services, 2002.

Faouzi Alaya Cheikh received his B.S. degree in electrical engineering in 1992 from École Nationale d'Ingénieurs de Tunis, Tunisia. He received his M.S. degree in electrical engineering (Major in Signal Processing) from Tampere University of Technology, Finland, in 1996. Mr. Alaya Cheikh is currently a Ph.D. candidate and works as a Researcher at the Institute of Signal Processing, Tampere University of Technology, Tampere, Finland. From 1994 to 1996, he was a Research Assistant at the Institute of Signal Processing, and from 1997 he has been a Researcher with the same institute. His research interests include nonlinear signal and image processing and analysis, pattern recognition and content-based analysis and retrieval. He has been an active member in many Finnish and European research projects among them Nobless esprit, COST 211 quat, and MUVI. He served as Associate Editor of the EURASIP Journal on Applied Signal Processing, Special Issue on Image Analysis for Multimedia Interactive Services. He serves as a reviewer to several conferences and journals. He co-authored over 30 publications. 
Bogdan Cramariuc received his M.S. degree in electrical engineering in 1993 from Polytechnica University of Bucharest, Faculty of Electronics and Telecommunications, Bucharest, Romania. Mr. Cramariuc is currently a Ph.D. candidate and works as Researcher for the Institute of Signal Processing at Tampere University of Technology, Tampere, Finland. From 1993 to 1994 he worked as Teaching Assistant at the Fac-

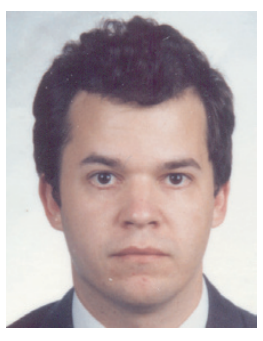
ulty of Electronics and Telecommunications at the Polytechnica University of Bucharest. During this period he has also been involved as Researcher with Electrostatica S.A., a national research institute in Bucharest, Romania. Since 1995 he has been with the Institute of Signal Processing at Tampere University of Technology, Tampere, Finland. His research interests include signal and image analysis, image segmentation, texture analysis, content-based indexing and retrieval in multimedia databases, mathematical morphology, computer vision, parallel processing, data mining, and artificial intelligence. Mr. Cramariuc has been an active member in several Finnish and European projects, such as Nobless, Esprit and MUVI. He served as Associate Editor of the EURASIP Journal on Applied Signal Processing, Special Issue on Image Analysis for Multimedia Interactive Services.

Mari Partio was born 1979 in Finland. Autumn 1998 she started her M.S. studies at Tampere University of Technology in department of Electric Engineering. She is majoring in Signal Processing and her minor is Software Engineering. Since summer 2000 she has been working as a research assistant at the Institute of Signal Processing. Her research interests include content-based image and video retrieval.

Pasi Reijonen was born 1976 in Finland. Started at Tampere University of Technology in Automation Degree Program 1996 and received M.S. degree 2001. Major: Electronics. Minor: Industrial Management, Automation and Control Engineering. For the academic year 1999-2000, he was as an Erasmus student in England (University of Leeds). Currently he is working as a researcher at Tampere University of Technology at Institute of Signal Processing. His research interests are Shape Analysis for Content-Based Image Retrieval.

Moncef Gabbouj received his B.S. degree in electrical engineering in 1985 from Oklahoma State University, Stillwater, and his M.S. and Ph.D. degrees in electrical engineering from Purdue University, West Lafayette, Indiana, in 1986 and 1989, respectively. Dr. Gabbouj is currently a professor and Head of the Institute of Signal Processing of Tampere University of Technology, Tampere, Finland. From 1995 to

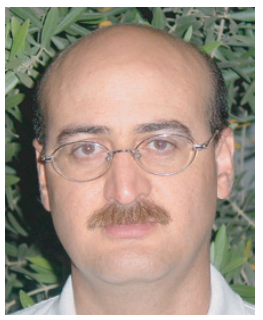
1998 he was a professor with the Department of Information Technology of Pori School of Technology and Economics, Pori, and during 1997 and 1998 he was on sabbatical leave with the Academy of Finland. From 1994 to 1995 he was an associate professor with the Signal Processing Laboratory of Tampere University of Technology, Tampere, Finland. From 1990 to 1993 he was a senior research scientist with the Research Institute for Information Technology, Tampere, Finland. His research interests include nonlinear signal and image processing and analysis, content-based analysis and retrieval and mathematical morphology. Dr. Gabbouj is the Vice-Chairman of the IEEE-EURASIP NSIP
(Nonlinear Signal and Image Processing) Board. He is currently the Technical Committee Chairman of the EC COST 211quat. He served as associate editor of the IEEE Transactions on Image Processing, and was guest editor of the European journal Signal Processing, special issue on nonlinear digital signal processing (August 1994). He is the chairman of the IEEE Finland Section and past chair of the IEEE Circuits and Systems Society, Technical Committee on Digital Signal Processing, and the IEEE SP/CAS Finland Chapter. He was also the TPC Chair of EUSIPCO 2000 and the DSP track chair of the 1996 IEEE ISCAS and the program chair of NORSIG' 96, and is the technical program chair of EUSIPCO 2000. He is also member of EURASIP AdCom. Dr. Gabbouj is the Director of the International University Program in Information Technology and member of the Council of the Department of Information Technology at Tampere University of Technology. He is also the Secretary of the International Advisory Board of Tampere International Center of Signal Processing, TICSP. He is a member of Eta Kappa $\mathrm{Nu}$, Phi Kappa Phi, IEEE SP and CAS societies. Dr. Gabbouj was co-recipient of the Myril B. Reed Best Paper Award from the 32nd Midwest Symposium on Circuits and Systems and co-recipient of the NORSIG 94 Best Paper Award from the 1994 Nordic Signal Processing Symposium. He is co-author of over 150 publications. Dr. Gabbouj was the prime investigator in two ESPRIT projects, 1 HCM project and several Tempus projects. He also served as Evaluator of IST proposals, and Auditor of a number of ACTS and IST projects on multimedia security, augmented and virtual reality, image and video signal processing. 